\title{
Larry Laudan: da noção de progresso científico à fundamentação do naturalismo normativo
}

\section{Larry Laudan: From the notion of scientific progress to the foundation of normative naturalism}

\author{
BEATRÍS DA SILVA SEUS ${ }^{1}$
}

\begin{abstract}
Resumo: No presente artigo, iremos analisar a proposta de uma normatividade inserida no modelo de uma visão de epistemologia naturalizada elaborada por Larry Laudan. O autor fundamenta uma regra que servirá de base metodológica para a inserção de descobertas científicas, além de criticar a noção antiga de racionalidade inserida nos escritos científicos do passado. Para tal, iremos salientar a necessidade de repensar certos tratados por um viés progressista.
\end{abstract}

Palavras-Chave: Epistemologia. Naturalismo. Laudan. Regras. Metodologia.

Abstract: In this article, we will analyze the proposal of a normativity inserted in the model of a vision of naturalized epistemology elaborated by Larry Laudan. The author bases a rule that will serve as a methodological basis for the insertion of scientific discoveries, besides criticizing the old notion of rationality inserted in the scientific writings of the past. To this end, we will stress the need to rethink certain treaties from a progressive perspective.

Keywords: Epistemology. Naturalism. Laudan. Rules. Methodology.

\section{Introdução}

No presente artigo, iremos ir de encontro ao naturalismo normativo de Larry Laudan, que busca retratar a história da ciência por um viés de análise do progresso, e não de sua racionalidade como havia sendo feito pelos historicistas de seu tempo. Para tal, Laudan irá analisar os contextos das mais variadas descobertas científicas, buscando evidenciar padrões metodológicos, trazendo luz à relação de meios e fins da pesquisa científica.

Após este primeiro momento, iremos analisar qual seria o critério para uma normatividade oriunda da tese da epistemologia naturalizada, tendo em vista a problemática da inexistência de juízos imutáveis dentro do campo científico. Assim sendo, demonstraremos como autores céticos como Feyerabens, Kuhn, Popper e Lakatos, estariam errados em adotar uma posição cética em relação à normatividade de uma teoria naturalista-epistemológica. Desta forma, Laudan irá devolver força prescritiva aos filósofos metodológicos contemporâneos, também evidenciando o erro de autores como Quine, Putman, Hacking e Rorty, que diziam que o papel dos cientistas naturalistas é puramente descritivo.

\footnotetext{
${ }^{1}$ Doutoranda pela Universidade Federal de Pelotas. Linha de pesquisa: Fundamentação e crítica da moral. E-mail: beatriseus@gmail.com
} 
Sendo assim, estaremos discutindo com Larry Laudan a possibilidade de trazer recursos técnicos e metodológicos para o campo da filosofia da ciência naturalizada, garantindo uma prescrição segura e um possível consenso entre metodologistas do século XXI: trabalho demasiado importante uma vez que temos diversos métodos rivais que podem vir a ser fundamentados no campo da Filosofia epistemológica; salientando uma noção de meta-metodologia como possibilidade de escolha de um método científico não-arbitrário.

\section{Distinção entre racionalidade e progresso científico}

Laudan afirma que apesar de vários filósofos estarem preocupados com a fundamentação de uma metodologia segura, que o empreendimento epistemológico tem sido descrito de forma prematura. Isto porque ele diz que têm sido abandonados diversos argumentos iniciais. Para combater um relativismo inserido na possibilidade de prescrições fruto da epistemologia naturalizada; Laudan irá promover a ideia de meta-metodologia.

Antes de entrar nessa questão, Laudan demonstra o argumento que ele considera ter sido o mais influente contra a meta-metodologia. Tal argumento está cercado do que ele chama de "historical turn" e esta frase se refere às visões dos autores como Kuhn, Feyerabend, Lakatos, Laudan, e Toulmin que durante os anos 6o e 70 argumentaram que nossa noção filosófica de racionalidade científica, falha em capturar a racionalidade das maiores descobertas científicas. Estes historicistas, chamados por Laudan, procuram demonstrar que grandes cientistas como Galileo, Newton, Darwin e Einstein contravieram todos os métodos científicos cânones que já foram fundamentados por filósofos. Laudan afirma que os historiadores de seu tempo insistem que existe uma falha de grandes cientistas em escolherem métodos de acordo com as recomendações filosóficas, pois quando o é feito, há uma redução da metodologia defendida, demonstrando a inadequação destas metodologias.

Laudan acredita que o requerimento de uma epistemologia ou metodologia que deva exibir e fundamentar a ciência antiga como racional é equivocada. Ele afirma ainda na introdução do artigo Progress or rationality? The prospects for normative naturalismo que nós podemos elaborar escolhas racionais e criteriosas entre teorias rivais de método ou de conhecimento sem insistir no requerimento racionalista. E justamente um dos propósitos do artigo trabalhado, é o de demonstrar que o requerimento de uma reconstrução racional não é nem desejada e nem necessária. O comentador Gonzáles, no artigo El pensamento de L. Laudan. Relaciones entre historia de la ciencia y filosofia de la ciencia salienta a distinção feita por Laudan entre uma filosofia da ciência, e uma história da ciência:

Aquí, em el análisis del problema de la normatividade y su relación com los enunciados empíricos, em el problema de la falácia 
naturalista, se encuentra a mi parecer, el núcleo del trabajo de Laudan em estas jornadas y centra buena parte de las discusiones que aparecen em el volumen. Si añadimos a ese núcleo temático la constante preocupación por parte de todos los autores para tratar de situarse em el complejo problema de la relación entre gilosogia de la ciencia e historia de la ciencia, podemos decir que este volumen puede ser uma buena ayuda para iniciarse em las discusiones contemporâneas em estos âmbitos de los estúdios sobre la ciencia (GONZÁLES, 1998, p. 95).

O comentador está se referindo ao trabalho de Laudan ao apontar que historiadores acreditam que a ciência tem sido uma atividade racional e que uma metodologia da ciência é por si mesma uma teoria da racionalidade. Nesta perspectiva, demonstra a teoria de que a metodologia é vista como uma teoria da racionalidade e que temos meios independentes e verificáveis de julgar a racionalidade de certos paradigmas de ações. A ideia de que podemos reconhecer certas ações ou episódios como racionais e cognitivos, independente de qualquer teoria sobre ações racionais, e que nós podemos usar estas ações como testes decisivos da adequação de uma teoria metodológica repousa numa intuição epistêmica que Laudan critica na página 20 do mesmo artigo citado anteriormente, onde afirma que "Here, however, I shall focus not on the blatant intuitionism of this position, but rather on its over-hasty identification of methodological soundness with rationality (LAUDAN, 1986, p. 20).

Para Laudan, é óbvio que os historicistas possuem uma estratégia de validar metodologias que requerem um comprometimento com a tese de que a ciência tem sido por excelência uma ação racional. Em suas visões, se uma metodologia promove visões de desacordos feitas por cientistas cânones; que então há evidência de que estes grandes cientistas não sejam irracionais, mas que a metodologia filosófica enquanto fundamento de suas teorias teria falhado em explicar a racionalidade. Neste campo, os filósofos entrariam em acordo com a afirmação de Lakatos de que: "A rationality theory [by which Lakatos specifically means a methodology]... is to be rejected if it is, inconsistent with na accepted 'basic value judgment' of the scientific elite" (LAUDAN, 1986, p. 20). A teoria da racionalidade (metodologia) será rejeitada se for inconsistente e com aceitação de um "julgamento valorativo básico" da elite científica.

O filósofo americano aponta que um conflito como este não é suficiente para rejeitar a metodologia, porém, os historicistas aqui trabalhados irão acordar com as escolhas da elite científica. Em cada caso, ninguém pergunta qual premissa rival a metodologia teria escolhido como mais segura, analisando suas evidências racionais. Em geral são comparadas as "teorias das escolhas" feitas por grandes cientistas, e não uma análise metodológica destas escolhas. Por isso Laudan afirma que se a metodologia nos leva a escolhas congruentes feitas por estes grandes nomes da ciência, então de acordo com os historicistas, teria sido exibida a racionalidade 
destas escolhas e teria sido estabelecidas suas próprias credenciais, uma fundamentação por si mesma. Se a metodologia adotada por Laudan leva a outras a preferencias diferentes das adotadas pela elite científica, então a metodologia deveria ser rejeitada pela afirmação errônea de que muitos grandes cientistas têm sido irracionais:

\begin{abstract}
Unfortunately, as I shall show shortly, both the assumption that a methodology has implications concerning the rationality of great scientists and the thesis that methodologies are to be evaluated in ligh of the degree to which they capture past science as rational are thoroughly wrongheaded (LAUDAN, 1986, p. 20).
\end{abstract}

Laudan demonstra que a afirmação de que a metodologia tem implicações referentes à racionalidade de grandes cientistas; e a tese de que as metodologias são validadas pelo grau em que elas captam a ciência passada como racionais são afirmações erradas. Isto ficará mais claro no próximo capítulo quando falarmos da distinção entre a tese da racionalidade e a tese da meta-metodologia.

\title{
A tese da meta-metodologia
}

Laudan salienta no artigo trabalhado duas doutrinas, uma denominada como "tese da racionalidade", e outra denominada como a "tese da meta-metodologia". Ambas as teses vão nos auxiliar na compreensão de mundo que Larry Laudan traz consigo ao abordar a história da ciência natural, e de que forma ele critica a concepção de que alguns autores seriam racionais ou irracionais à luz de uma concepção de mundo atual, ou seja, cometendo anacronismos:

\footnotetext{
As we have seen, the historicist subscribes to the following doctrines: the rationality thesis (RT): most great scientists have made their theory choices rationally. The meta-methodology thesis (MMT): a methodology of science is to be evaluated in terms of its ability to replicate the choices of past scientists as rational (LAUDAN, 1986, p. $20-21$ ).
}

Laudan afirma que de fato os historicistas estão certos em assumir que as metodologias existentes falham em fundamentar as teorias que a elite científica escolheu no passado. Por essa razão, autores como Kuhn e Feyerabend rejeitaram e tiveram como inadequadas todas as metodologias já elaboradas no campo da filosofia. Laudan demonstra seu propósito, portanto, de provar através de um exame, o porque de a metodologia e a racionalidade devem ser distinguidas e porque a meta metodologia é tão rechaçada:

I want to focus on just one of the howlers comitted by most of the historicists. Its examination will show why metohodology and rationality need to be sharply distinguised, and why MMT - the core meta-thesis os the historical school - is wholly unacceptable. What in particular has gone wrong is that the historicists' metamethodology has failed to reckon with the fact that both aims and 
background beliefs of scientists vary from agente to agente, and that this is particularly so when one if talking about scientific epochs very different from our own (LAUDAN, 1986, p. 21).

Para o autor, o problema foi que os historicistas meta-metodológicos falharam em reconhecer o fato de que tanto os objetivos, quanto os antecedentes das crenças dos cientistas variam de acordo com o agente, e que esta particularidade faz com que o discurso científico entre autores seja diferente. Se os objetivos dos cientistas variam com o tempo de forma significativa, Larry irá apontar que não podemos razoavelmente esperar que os métodos particulares de filosofia epistêmica sejam tomados, e nem que os cientistas queiram concluir aquilo que nós queremos concluir. Por isso o filósofo afirma que seja a racionalidade o que for não podemos esquecer que ela é uma relação entre agente e contexto específicos. Ou seja, Larru defende que quando se diz que um agente agiu racionalmente, estamos assumindo intrinsicamente que ele agiu de forma em que ele acreditou que iria alcançar seus fins desejados. Porém, agir de acordo com o desejo de um fim específico, não garante que a ação tenha sido racional; e filósofos irão discutir sobre este tópico. Porém, Laudan afirma que poucos irão discordar de que é uma condição necessária para que alguém seja racional, que este agente consiga determinar os meios necessários para atingir os fins desejados. Isto significa, que quando formos julgar a racionalidade de um agente, devemos considerar "What actions were taken; what the agent's ends or aims were; the background beliefs which informed his judgments about the likely consequences of his possible actions (LAUDAN, 1986, p. 21)." Para Laudan, não há uma concepção de racionalidade viável que não tenha estes pré-requisitos para determinar a racionalidade de um agente. Seu argumento é de que: se os cientistas do passado tinham objetivos diferentes dos nossos e crenças diferentes das nossas; então a racionalidade de suas escolhas ou ações não pode ser propriamente determinada pela pergunta "eles adotaram estratégias intencionadas aos nossos objetivos?". Assim o sendo, ele afirma que regras indicativas táticas e estratégicas dão designada para promover os objetivos de pesquisa de autores cânones. Portanto, seria em sua concepção apropriado utilizar nossos métodos para acessar a racionalidade de cientistas do passado apenas se suas utilidades cognitivas forem iguais às nossas, e também apenas se seus planos de fundo de crenças forem essencialmente iguais às do ponto de vista contemporâneo.

Laudan para deixar ainda mais clara a sua problematização, traz em seu texto uma pergunta que considera ser primordial: se os cientistas do passado tinham objetivos e crenças iguais às nossas; e para ele parece óbvio que não é o caso, mas sim que os cientistas do passado possuíam teorias a respeito do mundo e teorias causais diferentes entre si e diferentes das nossas. E mesmo que seus objetivos fossem similares aos nossos, seus conjuntos de crenças determinados pelos seus "backgrounds" os levariam a atribuir usos diferentes e cursos de ações diferentes daqueles que nós iríamos escolher. 
A situação é pior na medida em que está claro para Laudan que os cientistas do passado possuíam o que ele chama de uma "constelação de utilidades cognitivas" diferentes das nossas. Ou seja, Laudan está tentando nos demonstrar que o julgamento da teoria newtoniana como racional ou irracional, por exemplo, deve ser feito à luz de suas próprias convicções. Se assim não o for feito, não é possível defender ou não de forma segura a sua racionalidade, pois estaríamos fazendo uma análise anacrônica. Ou seja, Laudam defende que devemos, em uma perspectiva teórica, assegurar que o contexto (o naturalismo) dos autores aqui inseridos seja respeitado de forma a evitar afirmações equivocadas de sua relevância ou não. (nota 5).

The problem we have our finger on is simply this: to the extent that our judging na agent's having acted rationally involves taking his aims seriously, then methodologies designed to promote aims different from those of the agente appear incompetente to pronounce on the agent's rationality (LAUDAN, 1986, p. 22).

Ou seja, a ação de um agente, só pode ser julgada como racional ou não respeitando suas ferramentas cognitivas disponíveis. Para Laudan, esta análise faz com que seja possível salientar o que ele chama de fins genuínos da ciência. Além disso, o filósofo afirma que não é um problema que os agentes atinjam fins diferentes daqueles que motivaram suas ações (como o exemplo de um cientista que busca elaborar uma teoria de alguma coisa, e acaba comprovando outra através do uso de sua razão). De acordo com ele, é uma violência ir contra a usual noção de racionalidade só porque um cientista atingiu um fim que a princípio não estava englobado em seu objetivo inicial.

Toda esta análise é importante, pois seria inapropriado também julgar nossas metodologias como insuficientes apenas analisando se elas processam ações racionais de cientistas do passado. Isto porque nossos objetivos e planos de fundo são diferentes. Desta forma, racionalidade é uma coisa, na medida em que de fato estes cientistas aclamados foram sim racionais. Após aceitarmos que a autenticação de metodologias não está na asserção ou não delas nas teorias de cientistas do passado, é possível nos perguntarmos novamente como alguém pode garantir propostas metodológicas.

Primeiramente, Laudan busca olhar atentamente à epistemologia metodológica. Para ele, a metodologia consiste em um conjuno de regras ou máximas, partindo do mais geral ao mais específico. Regras típicas da metodologia incluem:

Propoud only falsiable theories; avoid ad hoc modifications; prefer theories which make successful surprising predictions over theories which explain only what is already known; when experimenting on human subjects, use blinded experimental techniques; reject 
theories which fail to exhibit an analogy with succesful theories in other domains; avoid theories which postulate unobservable entities; use controlled experiments for testing causal hypotheses; reject inconsistents theories; prefer simple theories to complex ones; accept a new theory only if it can explain all the successes of its predecessor (LAUDAN, 1986, p. 23).

Todas estas regras, de acordo com Laudan, foram postuladas por filósofos metodológicos contemporâneos. E a pergunta chave da meta-metodologia é como prover um mandado para rejeitar ou aceitar tais regras metodológicas. Deve-se ressaltar que essas regras ou máximas possuem a forma de comandos na perspectiva do autor. Neste sentido, elas não podem ser consideradas verdadeiras ou falsas, mas sim úteis ou inúteis. Para Laudan, todas as regras metodológicas não devem ser construídas como num modelo de imperativo categórico, mas como imperativos hipotéticos ${ }^{2}$. Ou seja, antecedendo objetivos ou expressões de finalidade de uma ação. Desta forma, seria possível escolher entre metodologias rivais mais facilmente do que entre teorias rivais. Alexander Rosenberg no trabalho intitulado de Normative naturalismo and the role of philosophy, esclarece o motivo de Laudan defender o uso dos imperativos hipotéticos, no lugar dos imperativos categóricos em seu trabalho, referindo-se à todas aquelas regras propostas pelo filósofo americano na fundamentação de uma normatividade metodológica:

Despite appearances, these rules are not categorical imperatives; they are hypothetical imperatives with suppressed antecedentes, and they all have the same suppressed antecedentes (...) These hypothetical imperatives do not specify the goals of science, they do not even require that science has a small number of identifiable goals on which all scientists, past and presente, agree (ROSENBERG, 1988, p. 350).

Retirando as possibilidades de apriorismos em seu trabalho, neste ponto, Laudan preocupa-se também em distanciar-se do naturalismo de Quine3: ele afirma que Quine e outros naturalistas quineanos, acreditavam que a epistemologia deveria ser inteiramente não normativa, mas sim um punhado de descrições psicológicas, onde nos concentraríamos em reconstruir a espinha dorsal do conhecimento que hoje chamamos de ciência. Porém, para o autor a desnaturalização da metodologia não é implicada por sua naturalização. Muito pelo contrário, pode-se mostrar que

\footnotetext{
${ }^{2}$ Na página 24 do artigo estudado, podemos observar alguns exemplos disto.

3 Rodolfo Gaeta e Susana Lucero, no artigo intitulado de Naturalismo, normatividade y racionalidade em Laudan, pontuam esta diferença: "A diferencia de la propuesta de Quine - para quien la naturalización de la epistemologia la transforma em uma rama de la psicologia - Laudan sostiene que su naturalismo no implica la pérdida del caráter prescriptivo. No encontramos em su obra fundamentos para esta afirmación, aunque evidentemente la normatividade está implícita em la forma imperativo-contingente que adoptan las reglas metodológicas (GAETA; LUCERO, 2003, p. 164).
} 
uma metodologia "científica" e robustamente "descritiva" completa terá conseqüências normativas.

\section{Critérios para um naturalismo normativo}

Para mostrar aos leitores a adequação das regras do teste metodológico, é necessário demonstrar estas regras no contexto descritivo de afirmações teóricas. Como podemos observar na nota 6, Laudan demonstrar como o debate entre metodologistas pode "re-cast" (re-elenco) como declarações contingentes podem ser feitas entre fins e meios. De acordo com o autor, isto não nos diz como especificamente estas regras metodológicas irão ser testadas, mas apenas demonstrar insistentemente como as regras instrumentais da empiria não possuem apenas um status de problematização. Nesta perspectiva, escolher entre teorias metodológicas rivais (chamadas por ele de famílias de regras metodológicas) é tão problemático quanto escolher entre teorias empíricas rivais de qualquer área do saber. Ou seja, o caráter imperativo da escolha de regras metodológicas não as fazem estar em um patamar diferenciado de descrições da realidade. Conforme os intérpretes Gaeta e Lucero, o objeto perseguido por Laudan é o seguinte:

El proyecto de edificar uma teoria del método naturalista y normativa tiene méritos y desventajas, entre los primeiros está el propósito manifiesto de excluir concepciones marcadamente aprioristas así como la pretensión arrogante de indicar a los científicos como deben proceder em su tarea específica (...) El naturalismo de Laudan se propone además desvincular totalmente la racionalidade del método y del progresso pero lo logrea efetivamente? Su modelo se basa en postular reglas cuya estructura y semântica es la de um enunciado hipotético que afirma una relación contingente entre medios y fines (GAETA; LUCERO, 2003, p. 165).

Laudan ressalta que tendo estes elementos de meio e fim em vista, deve-se ainda ter em mente, que para que possamos testar uma regra metodológica, devemos antes estabelecer uma outra regra metodológica primeira, que nos dirá como testar a primeira. E em seguida, esta outra regra também precisará de uma nova para fundamentar-se. Isso é chamado por ele de circularidade viciosa de regresso infinito. Para quebrar este ciclo, ele responde que podemos dissolver o regresso na medida em que se consiga encontrar um princípio de evidencia que todas as teorias metodológicas possuem em comum. Se este princípio existir, então ele pode ser invocado como neutro e imparcial na escolha de regras metodológicas rivais. Porém, devemos nos perguntar se apesar de todas as teorias de metodologia científicas possuem este princípio neutro, tendo em vista todas as suas particularidades e peculiaridades: se elas compartilharem entre si princípios empíricos de suporte, eles poderão ser descritos como incontroversos. 
Para Laudan, este critério de escolha está presente em nossas convicções indutivas sobre estratégias. Eis a regra:

$\left(\mathrm{R}^{1}\right)$ If actions of a particular sort, $\mathrm{m}$, have consistently promoted certain cognitive ends, e, in the past, and rival actions, n, have failed to do so, then assume that future actions following the rule "if your ai mis e, you ought to do m" are more likely to promote those ends than actions based on the rule "if your ai mis e, you ought to do n" (LAUDAN, 1986, p. 25). ${ }^{4}$

Para Laudan, esta regra não sofisticada ou interessante para a escolha entre estratégias rivais de pesquisa. Para ele, objetivo de uma teoria formal da metodologia é desenvolver e garantir o critério mais complexo e seguro de suporte evidencial. Devemos nos perguntar se a regra acima é assumida de forma universal por todos os filósofos da ciência e se ela independentemente do consenso entre autores, parece ser uma regra de aprendizagem a partir da experiência. Se assim não o for, ela não servirá de critério. Por razões como essa, Laudan afirma que é plausível dizer que haveria consenso filosófico científico sobre a apropriação de pressupostos como da regra descrita acima.

Mesmo que se afirma que $\mathrm{R}^{1}$ seja consenso entre metodologistas contemporâneos, como partir dessa regra para uma solução meta-metodológica para nossos enigmas? Para Laudan, os passos são simples: primeiro deve-se assumir que $\mathrm{R}^{1}$ é algo dado racionalmente. Deve-se assumir, em seguida, que afirmamos todas as regras metodológicas numa relação de meios e fins. Nossa tarefa, em seguida, será:

To ascertain whether, for any proposed methodological rule, there is evidence (of the sort countenanced by $\left(R^{1}\right)$ ) for the assertion of the co-variance postulated by the rule. If the answer to that question is affirmative, then we have shown the warrant fpr accepting that rule rather than any of its (extant) rivals. If the answer is negative, then we have grounds for rejecting the rule (LAUDAN, 1986, p. 26)

Laudan afirma que se conseguirmos evidência de que seguir uma certa regra promove nossos fins melhor do que qualquer outra regra, então que temos fundamento para endossar esta regra. Se tivermos evidencia de que agindo de acordo com a regra teremos frustrado a realização de nosso fim cognitivo, então temos fundamento para rejeitar a regra. O filósofo ainda chama a atenção para notarmos que questões sobre a racionalidade ou irracionalidade de episódios

\footnotetext{
4 Tradução nossa: "Se ações de um tipo particular, m, tiverem promovido consistentemente certos fins cognitivos, e, no passado, e ações rivais, n, falharam em fazê-lo, então suponha que ações futuras seguindo a regra 'se você apontar é e, você deve fazer m são mais propensos a promover esses fins do que ações baseadas na regra "se o seu objetivo é e, você deve fazer para n'."
} 
particulares na história da ciência. Devemos nos perguntar apenas sobre quais métodos foram promovidos, ou que tenham falhado na hora de terem sido promovidos, nas necessidades de fins cognitivos no passado. Para ele, algumas vezes será fácil responder esta questão, outras vezes não, mas é uma questão empírica importante.

Para Laudan, conforme ele propõe uma normatividade inserida na epistemologia naturalizada, ele propõe também certa estratégia de pesquisa que evitando modificações ad hoc, produz teorias que tem como consequência uma plausabilidade na medida em que há possibilidades de testes. Normalmente, este debate entre filósofos teria como problema as intuições metodológicas. Para saber qual lado defender entre os autores que assumem premissas ad hoc em seus trabalhos e os que fazem o contrário, Laudan se pergunta no texto se teorias com o elemento hac hoc tem provido ou não menos conhecimento do que as que não possuem o mesmo elemento. Para ele, ainda não teríamos esta resposta. A grande virada para esta pergunta, conforme o autor, é demonstrar que os problemas da meta-metodologia e das regras metodológicas tem um caráter diferenciado de afirmações empíricas ordinárias, sendo então promovidas por um processo de pensamento diferenciado. Em sua opinião, o propósito da metodologia é descobrir as estratégias mais efetivas para a investigação do mundo empírico. Essa pesquisa pode ou não nos envolver em articular critérios para a validação usada por cientistas do passado ${ }^{5}$.

Os naturalistas meta-metodológicos, conforme descritos por Laudan, não precisam de intuições pré-analíticas sobre casos, ou informações sobre escolhas da elite científica, nem concepções a priori sobre quais disciplinas são científicas ou não. O que precisamos na meta-metodologia segundo ele, são dados sobre quais estratégias de investigação tendem a promover quais fins cognitivos. Por isso Laudan tem dito que o fundamental para a problemática da metodologia é provar a evidência sobre a conexão entre meios e fins. Isto não tem relação a explica a racionalidade ou irracionalidade de pensadores do passado. $O$ que precisa ser explanado, é que a ciência tem sido bem sucedida na produção de material epistêmico. Nós levamos a ciência a sério, de acordo com ele, porque ela tem promovido fins que consideramos serem cognitivamente importantes. Ou melhor, com o passar do tempo, ela tem apresentado um progresso prático. Diferentemente da racionalidade, o progresso não precisa de uma noção específica dos agentes envolvidos. Podemos, portanto, falar sem cair em contradição, acerca de uma certa sequencia de eventos que representam progresso científico:

Hence, the history of science has to be reckoned with, not because scientists are always or more often rational than anyonse else (...), but rather because the history os science - unlike that of many

\footnotetext{
${ }^{5}$ A grande tarefa é muito mais de encontrar meios para um fim, do que o fim em si mesmo.
} 
other disciplines - offers an impressive record of actions and decisions moving closer through time to a realization of ends that most of us hold to be important and worthwhile. The record that is the history of science shows us what sorts of cognitive ambitions have been realized and what sorts have not. If we were today espousing cognitive aims which had not been progressively realized in the development of science, then that history would put few constraints on our methodological musings (LAUDAN, 1986, p. 28).

Nestas circunstancias, Laudan defende que não tomemos a metodologia de forma a priori $^{6}$. Apesar de não podermos ignorar o grande número de trabalhos efetivados com sucesso, tendo premissas a priori como fundamento, Laudan defende poder haver também sucesso naqueles trabalhos teóricos que tem usado determinados métodos que defendam o apriorismo. Neste sentido, para Laudan, as ciências nos oferecem estratégias implicitas que demonstraram serem bem sucedidas ao promover os fins desejados, mas salienta que este viés argumentativo também precariza o trabalho dos filósofos, que podem partir de postulações metafísicas. Desta forma, Laudan defende um tipo de moderação entre pesquisas empíricas e defesas de valores que não podem ser observados puramente empiricamente, mas podem ser defendidos através da experiência. Nesta análise, a construção de uma metodologia cientifica se deve ao desenvolvimento de diversas regras metodológicas, concebidas como imperativos hipotéticos, que promovem fins epistemicos e cognitivos. E assim a metodologia se detém em articular meios e fins.

\section{Considerações finais}

Em suma, Laudan demonstrou com o texto trabalhado, a necessidade de não fazermos uso de visões anacronistas no momento de executar uma análise acerca de quaisquer tipos de tratados, sejam eles científicos, ou da filosofia da ciência. Surge com o autor a necessária vinculação de análise entre meios, fins e contextos de autores a serem estudados, possibilitando não mais uma visão racionalista da história científica, mas salientando a problemática do progresso, que define a ciência até os dias atuais.

Ao situar certas regras importantes para uma metodologia científica segura, evitando um regresso ao infinito ao postular uma regra primeira, o autor identifica a possibilidade de investigações por parte da filosofia epistemológica partindo de uma noção axiológica do saber. Isto é, quando um determinado autor, filósofo, cientista e etc busca por executar uma investigação, ele indiretamente está postulando um determinado tipo de valor a ser comprovado. Nesta perspectiva, estes tipos de pesquisas não conseguem desvincular-se de proposições axiológicas, possibilitando

\footnotetext{
${ }^{6}$ Como podemos também perceber na conclusão de seu artigo, quando diz: "What it does not promise is any a priori or incorrigible demonstrations of methodology; to the contrary, it makes methodology every bit as precarious epistemically as science itself (LAUDAN, 1986, p. 29)."
} 
então não só a refutação da teoria de Quine (por exemplo) que defenderia uma filosofia puramente descritiva, como também defende a possibilidade de inserir a questão da normatividade nestas pesquisas científicas.

Independentemente de possíveis acidentes históricos inseridos na história da pesquisa humana, Laudan sempre salienta em seu trabalho que a importância da pesquisa está em seus resultados, e seu naturalismo normativo, desta forma, é uma meta-metodologia na medida em que consegue constituir um método que seja empírico ao mesmo tempo que é normativo, articulando provas com critérios de validação metodológica.

\section{Referências}

LAUDAN, Larry. Progress or rationality? The prospects for normative naturalism. American Philosophical Quarterly. Volume 24, Number 1: 1987.

GAETA; LUCERO. Naturalismo, normatividad y racionalidad en Laudan. Epistemologia e Historia de la Ciencia, vol. 9, no 9: 2003.

GONZÁLEZ, Wenceslao. El pensamiento de L. Laudan. Relaciones entre historia de la ciencia y filosofia de la ciencia. Revista Hispanoamericana de Filosofia. Volume XXXI, noo 92: 1999.

GUILLAUMIN, Godfrey. El naturalismo normativo y sus problemas (normativos). Signos filosóficos, vol. X, no 20: 2008.

ROSENBERG, Alexander. Normative naturalism and the role of philosophy. Philosophy of science, vol. 57, no 1: 1990.

Submissão: 14. 10. 2019 / Aceite: 15. 04. 2020 